Gut, 1971, 12, 756-772

\title{
Supplement
}

\section{European Association for the Study of the Liver}

The 6th meeting was held in London on 2, 3, and 4 September under the presidency of B. H. Billing (United Kingdom). The following are the abstracts of the first 36 papers. Altogether 157 abstracts were submitted but only those which were read at the meeting are reprinted in Gut. The remainder can be found in the booklet of abstracts which each member received at the meeting.

The conference of the EASL was preceded by a meeting of the Liver Club.

1

\section{Influence of Theophylline on Canalicular Bile Production in the Dog}

S. ERLINGER and M. DUMONT

Unité de Recherches de Physiopathologie

Hépatique,

Hôpital Beaujon,

92-Clichy, France

Recent experimental reports support the hypothesis that a large fraction of hepatocytic bile is not dependent on bile salt excretion. However, the mechanism of elaboration of this fraction is not established. Suppression of this bile salt-independent flow in the rabbit by inhibitors of sodium transport suggests that it may be the result of active sodium transport by the hepatocytes. These studies were undertaken to examine the influence on bile formation in the dog of theophylline, an agent known to stimulate sodium transport.

In ten anaesthetized dogs, the bile duct was cannulated, a bile salt infusion ( $6 \mu \mathrm{moles} / \mathrm{min})$ was given to obtain a stable bile flow. (1) Theophylline $(280 \mu$ moles followed by a constant infusion of $20 \mu \mathrm{moles} / \mathrm{min}$ ) produced a significant increase in bile flow, from an average control value of $0.165 \pm$ (1 SD) $0.070 \mathrm{ml} / \mathrm{min}$ to $0.294 \pm 0.073 \mathrm{ml} / \mathrm{min}$ $(\mathrm{P}<0.005)$. (2) Bile salt concentration in bile decreased significantly from $55.9 \pm 22.9$ mmoles $/ 1$ to $30.4 \pm 14.7 \mathrm{mmoles} / \mathrm{l}(\mathrm{P}<0.005)$. (3) Bile salt excretion rate was not significantly modified.
(4) ${ }^{14} \mathrm{C}$-erythritol clearance increased in proportion to bile flow, indicating that choleresis was of hepatocellular rather than ductular origin. (5) ${ }^{3} \mathrm{H}$-labelled theophylline, given intravenously, was excreted in bile and allowed quantitative estimation of the amount of theophylline recovered in bile: in two dogs, during the 80 minutes following an injection of $560 \mu$ moles of theophylline, the mean biliary excretion of the drug was 0.09 and $0.12 \mu \mathrm{moles} / \mathrm{min}$, an amount too small to produce an osmotic choleresis. (6) Sodium excretion in bile increased and a linear relationship was obtained between sodium excretion and bile flow.

These studies support the postulate that, in the $\mathrm{dog}$, the bile salt independent fraction of bile could be the result of active sodium transport by the hepatocytes.

2

Effect of Indocyanine Green on Bile Flow and Bile Salt Excretion. A Model to Study Cholestasis

W. HORAK, G. GRABNER, and G. PAUMGARTNER

2nd Medical University Clinic of Vienna, Austria, and Department of Clinical Pharmacology, University of Berne, Switzerland

Biliary secretion of organic anions generally leads to 756 
an increase in bile flow. Under certain conditions, however, several anionic dyes including indocyanine green (ICG) inhibit bile flow. Elucidation of the underlying mechanism may add to the understanding of the pathophysiology of cholestasis.

Bile flow and biliary excretion of bile salts (BS) and ICG were measured during constant i.v. infusion of ICG in male Wistar rats. BS lost due to interruption of enterohepatic circulation were continuously replaced by i.v. infusion of sodium taurocholate. BS were determined enzymatically.

ICG infusions of 10 and $20 \mathrm{nmoles} / \mathrm{min} / 100 \mathrm{~g}$ body weight, which were both below the transport maximum (Tm) of ICG, had no significant effect on bile flow and BS excretion. However, during infusions of 40 and $80 \mathrm{nmoles} / \mathrm{min} / 100 \mathrm{~g}$, which were above the $\mathrm{Tm}$, bile flow diminished rapidly reaching $80 \%$ and $56 \%$ respectively of preinfusion values at 40 minutes. Simultaneously BS output decreased to $93 \%$ and $84 \%$ only, resulting in an increase in BS concentration in bile. Single injections of large doses of ICG $(3 / \mu$ moles $/ 100 \mathrm{~g})$ had similar effects. The decrease in bile flow was not related to biliary ICG output but rather to ICG blood levels and to the estimated accumulation of ICG within the liver. It could not be explained by the observed decrease in BS secretion. These data suggest that ICG mainly inhibits the formation of a BS independent fraction of bile. Thus, ICG cholestasis may be a useful model to study drug induced cholestasis.

3

\section{Metabolism and Choleretic Effect of Dehydrocholate in the Dog}

\author{
J-F. DESJEUX, S. ERLINGER, and \\ M. DUMONT \\ Unité de Recherches de Physiopathologie \\ Hépatique, \\ Hôpital Beaujon, \\ 92-Clichy, France
}

Although early studies have established the choleretic effect of the synthetic, triketo 'bile acid' dehydrocholate (DHC), the metabolism of this agent and its influence on hepatic excretory function are poorly understood. Because it is water insoluble and does not form micelles in vitro, it can be predicted that it has to be transformed prior to its excretion into bile. These studies were undertaken to answer the following questions: (a) what are the metabolites of DHC in the dog? (b) Can these metabolites form micelles and enhance phospholipid and cholesterol excretion? (c) Is their choleretic effect different from that of taurocholate (TC)?

Equimolar infusions of DHC and TC were given intravenously to eight anaesthetized dogs and one unanaesthetized dog with a Thomas duodenal cannula. Bile was analysed by enzymatic, thin-layer chromatographic and ultracentrifugation techniques.

It was observed that: (1) approximately $50 \%$ of the DHC infused was excreted into bile as metabolites which reacted with the 3-hydroxysteroiddehydrogenase, indicating that it had been hydroxylated in the 3-position during its passage through the liver. (2) Isopycnic gradient ultracentrifugation of bile collected during DHC infusions allowed isolation of these hydroxylated metabolites, suggesting that they had formed a macromolecular complex. (3) Nevertheless, phospholipid and cholesterol excretions in bile were significantly lower during DHC infusions (respectively $1.66 \pm 0.79 \mathrm{mg} / \mathrm{min}$ and $21 \pm 34 \mu \mathrm{g} / \mathrm{min}$, mean $\pm 1 \mathrm{SD}$ ) than during TC infusions (respectively $9.35 \pm 3.71 \mathrm{mg} / \mathrm{min}$ and $287 \pm 253 \mu \mathrm{g} / \mathrm{min})(\mathrm{P}<0.01)$. (4) Bile flow was significantly higher during DHC infusions than during TC infusions $(P<0.01)$. (5) Unchanged DHC was not detected in bile by thin-layer chromatography during DHC infusions.

In conclusion, in the dog: (1) DHC is partly excreted in bile as 3-hydroxylated metabolites; (2) although apparently present in bile as a macromolecular complex, these metabolites do not enhance phospholipid and cholesterol excretion; (3) choleresis is greater during DHC infusions than during TC infusions; this is not due to the appearance in bile of unchanged DHC.

4

\section{Secretin and Bile Salt Induced Choleresis in Patients with and without Diseases of the Liver and Biliary Tract}

Ch. BODE, H. O. NEUBERGER, H. GOEBELL, and O. ZELDER

Departments of Internal Medicine and Surgery, University of Marburg, D-355 Marburg/L, Germany

In the present study the effect of secretin and bile salt infusions on bile flow and bile composition was investigated in $(a)$ patients with normal liver function and histological findings, $(b)$ patients with biochem- 
ical and/or histological signs of cholestasis, and (c) in patients with liver cirrhosis.

To study bile production quantitatively a balloonequipped $T$. tube was placed into the common bile duct in patients undergoing biliary surgery. Three to four days after the operation the bile was repeatedly collected in each patient for 2-8 hrs during intravenous infusion of different doses of secretin (Boots, Nottingham) and/or dehydrocholate and orally infused taurocholate.

\section{Results}

SECRETIN INFUSION

Maximal stimulation of bile flow was observed in all three groups following $8 \mathrm{clin}$. U/kg body weight/h of secretin. Stimulation of bile flow induced by smaller doses of secretin (1 clin. U/ kg body weight/h) was strikingly more pronounced in $(b)(2-5$-fold) and (c) (5-7-fold) as compared to (a). Similar differences were found under maximal stimulation. The output of bicarbonate and chloride during secretin application correlated closely in $(a)(r=0.98)$, while in $(b)$ and $(c)$ the percentage increase of bicarbonate exceeded distinctly that of chloride. Contrary to the concentration of $\mathrm{Na}^{+}$and $\mathrm{K}^{+}$the concentration of $\mathrm{Ca}^{++}$in the bile markedly decreased following secretin and thus resembled the behaviour of bilirubin and bile salts. This suggests that $\mathrm{Ca}^{++}$is secreted at a separate place (hepatocyte?).

\section{BILE SALT INFUSION}

When taurocholate was administered the 'extra output' of bile salts showed a good correlation with biliary water excretion $(r=0 \cdot 89)$. Bile salt induced choleresis did not differ significantly in $(b)$ and $(c)$ as against $(a)$. With increasing bile salt output a nearly equimolar fall in chloride output occurred. Dehydrocholate did not have a greater choleretic effect than taurocholate, probably because it is transformed into micelle forming derivatives as has been demonstrated earlier in animal experiments. In $(b)$ and $(c)$ dehydrocholate transformation is reduced as compared to (a). Dehydrocholate infusion $(0.4 \mathrm{~g} / 10 \mathrm{~kg} / \mathrm{h})$ reduced bilirubin excretion by $70-80 \%$. This effect points to a possible competition of both substances for excretion by the liver cell.

The results demonstrate that diseases of the liver and biliary system affect especially the bile salt independent fraction of bile flow, while the bile salt dependent secretion is less influenced.

\section{5}

\section{Hepatic Clearance and Biliary Excretion of Unconjugated Dyes in the Dubin-Johnson Syndrome}

\author{
J-F. DESJEUX, D. DHUMEAUX, \\ S. ERLINGER, and J-P. BENHAMOU \\ Unité de Recherches de Physiopathologie Hépatique \\ Hôpital Beaujon, F92-Clichy, and \\ INSERM U 99, \\ Hôpital Henri Mondor, F94-Créteil, France
}

The secondary rise in plasma concentration of bromsulfalein (BSP) after a single injection, in patients with the Dubin-Johnson syndrome, is not clearly understood. The purpose of this study was to test the hypothesis that this rise is related to the conjugation of the dye. In two patients with the Dubin-Johnson syndrome, the plasma disappearance curve of BSP was compared to that of three dyes which are not conjugated prior to their excretion into bile: indocyanine green (ICG), dibromsulfalein (DBSP) and ${ }^{125} \mathrm{I}$-rose bengal $\left({ }^{125} \mathrm{I}-\mathrm{RB}\right)$. In the two patients, it was observed that: (1) the initial plasma disappearance rates (from 0 to $20 \mathrm{~min}$ ) were normal for the four compounds; (2) plasma concentration of BSP increased after $45 \mathrm{~min}$; (3) in contrast, plasma concentration of ICG, DBSP and ${ }^{125}$ I-RB did not increase, up to $150 \mathrm{~min}$, a finding already observed by others for ICG.

In one of these patients with a biliary fistula, the biliary excretion of BSP and DBSP were measured. The percentage of the injected dose $(350 \mu$ moles $)$ recovered in bile during the three hours following the injection was similar for the two compounds: $5.4 \%$ for BSP and $5.2 \%$ for DBSP, suggesting that the biliary excretion of the two dyes was similarly impaired.

It is concluded that: (1) the secondary rise in plasma concentration of dyes in the Dubin-Johnson syndrome is not observed with the dyes which are not conjugated by the liver; (2) this fact is not the result of a greater biliary excretion, since biliary excretion of DBSP was impaired as well as that of BSP. It is postulated that the secondary rise in plasma concentration of BSP is due to re-entry of conjugated BSP into the plasma compartment as a result of a looser binding of the conjugate to liver cell constituents. 
Laboratorium Fysiopathologie, Academisch Ziekenhuis Sint-Raphaël,

Electron Microscopy and Histochemistry of Canalicular Differentiation in Fetal and Neonatal Rats

\author{
C. DE WOLF-PEETERS, R. DE VOS, and \\ V. J. DESMET \\ Laboratorium voor Histochemie en Cytochemie \\ Dienst Pathologische Ontleedkunde B \\ Academisch Ziekenhuis St. Rafaël \\ B 3000 Leuven, Belgium
}

Electron microscopic and histochemical investigations were carried out on the differentiation of the biliary pole of the hepatocyte in fetal and neo-natal rat liver.

During canalicular development, four successive types can be distinguished morphologically.

Type 1 (found mainly at 16-17 days of fetal life) corresponds to a primitive canalicular structure, without real lumen, formed by intracytoplasmic invaginations of the cell membrane between two junctional complexes. At 19-20 days, most canaliculi correspond to type 2, showing an irregular lumen, formed by sequestration of cytoplasmic fragments. Just before and after birth, most canaliculi are of type 3, characterized by a wide and regular lumen, devoid of microvilli. From the second day of life, an increasing number of canaliculi acquire microvilli and gradually correspond to type 4 , which has a normal adult morphology.

Histochemical results of alkaline phosphatase and adenosine triphosphatase at optical and ultrastructural level are in complete agreement with the described morphological maturation sequence, and further correlate with histochemical alterations known to coincide with abnormal canalicular morphology in pathological conditions of the adult.

It is suggested that the morphological and histochemical characteristics of immature canaliculi of type 3 in the perinatal period, which show striking similarity to 'cholestatic features' in the adult, are the expression of a secretory insufficiency of the neonatal liver cell.

Enzymic Formation of Bilirubinglucoside and Bilirubinxyloside

\author{
J. FEVERY, K. P. M. HEIRWEGH, \\ F. COMPERNOLLE, and J. DE GROOTE
}

3000 , Leuven, Belgium

Although glucose conjugation was thought to be important only in plants, bacteria and insects, bilirubin conjugated with glucose or xylose has been detected in mammals.

Bilirubin was coupled with diazotized ethylanthranilate and the azopigments separated in successive thin layer chromatography (t.l.c.) systems. The conjugating moiety was identified as glucose or xylose by t.l.c. against reference sugars, by mass spectrometry and by enzymic hydrolysis ( $\beta$-glucosidase). The glucose conjugate represented 11 to $30 \%$ of total conjugates in dog, cat, rabbit and chicken bile and 5 to $8 \%$ in human bile; the xylose conjugate $10 \%$ in dog bile, $6 \%$ in rabbit bile, $2 \%$ in human bile and trace amounts in the other species.

The enzymic synthesis of these new conjugates was obtained in liver cell homogenates or microsomal fractions of rat, dog and human liver incubated with unconjugated bilirubin and UDP-glucose or UDPxylose. It could be demonstrated by the study of the azopigments that these bilirubin conjugates consist of bilirubin monoglucoside and mixtures of monoglycoside and dixyloside. Enzymic kinetics were studied for glucosyl- and xylosyltransferase and compared to the glucuronyltransferase. At lower $p \mathrm{H}$ levels more dixyloside was formed and at $\mathrm{pH} \mathbf{7 \cdot 4}$ mainly the monoxyloside. With UDP-glucuronic acid and UDP-glucose only monoconjugates could be obtained at the different $p \mathrm{H}$-levels.

Gunn rats, which are known to be deficient in glucuronyltransferase are also deficient in these new conjugating enzymes.

8

\section{A Chemical Proof for the Existence of Bilirubin Monoglucuronide}

\author{
F. H. JANSEN and B. H. BILLING \\ Department of Medicine, \\ The Royal Free Hospital, \\ London, WC1X 8LF
}

Bilirubin is excreted as esters of glucuronic acid and acidic disaccharides. Theoretically both diconjugates and monoconjugates could be formed. No conclusive evidence for the existence of monoconjugates has, however, been presented and there is no information 
as to which side-chain of bilirubin is involved in monoconjugation. Preparations of bile pigments (Lucassen) were treated with concentrated ammonia resulting in the formation of bilirubin amides by nucleophilic substitution at the carbonyl carbon of the esterified propionic acid side chain. The amides were separated by t.l.c. and identified by mass spectrometry and accurate mass measurements. From bile pigment samples containing predominantly diconjugates (as suggested by the ethyl anthranilate azopigment technique) the main product was bilirubin diamide. From preparations containing largely monoconjugates the major products were two monoamides in approximately equal amounts. The exact isomeric composition of these mono-amides was established by studies of their azopigment derivatives. In view of the specificity of the reaction and the nature of the glycosides involved in conjugation it is possible to conclude that bilirubin monoconjugates are excreted in bile in two isomeric forms and in particular that bilirubin monoglucuronide exists as a chemical entity.

\section{9}

\section{Glucuronyl Transferase and Beta-Glucuronidase Activity Assayed with Different Substrates in Human Liver Disease and in Gilbert's Syndrome}

\author{
L. OKOLICSANYI, J. FREI, P. MAGNENAT, \\ and R. NACCARATO, with the technical \\ assistance of HEIDI BIRCHMEIER \\ Istituto Patologia Speciale Medica, University of \\ Padua, Italy \\ Central Laboratory of Clinical Chemistry and \\ Department of Medicine, Cantonal Hospital, \\ Lausanne, Switzerland
}

Liver-GT activity was assayed with different substrates in tissue samples obtained by needle biopsy from 111 patients with various liver diseases. In 18 cases with Gilbert's and in five with CriglerNajjar's syndrome a significantly decreased activity of GT was observed only with bilirubin as acceptor, while with 4-MU and PNP normal or slightly reduced values were found. In all other patients GT activity assayed with the three substrates was different and no statistically significant correlations were found between the obtained values. These results suggest the existence of at least two GTsystems. Moreover, it is suggested, that in Gilbert's and in Crigler-Najjar's syndrome, the activity of only one of these systems would be deficient, while the other(s) maintain normal functions. In the same tissue specimens liver Beta-G activity was determinated with two substrates. Slight variations of the mean values were observed, when compared to normals. Finally, it was not possible to demonstrate a linear correlation between the variations of the two systems: GT and Beta-G, when statistical analysis was carried out on each group or on the total case material.

\begin{tabular}{ll}
\multicolumn{1}{c}{ Abbreviations } \\
GT & UDP-glucuronyl transferase (EC 2.4.1.17) \\
4-MU & 4-methyl umbelliferone \\
PNP & p-nitrophenol \\
Beta-G & Beta-glucuronidase (EC 3.2.1.31).
\end{tabular}

10

The Cytochrome P-450 Content and the Activities of Various Microsomal Enzymes in Liver Biopsy Material from Patients

\author{
B. SCHOENE, R. A. FLEISCHMANN, \\ H. REMMER, and H-F. v. OLDERSHAUSEN \\ Institut für Toxikologie und Medizinische \\ Universitätsklinik, Tübingen, Germany
}

Micromethods allow the investigation of various, particularly drug-metabolizing, microsomal enzymes in needle biopsy material from human liver.

Because of the small amount of tissue available we used liver homogenate as enzyme source for measuring the amount of cytochrome $\mathrm{P}-450$ and the activity of the following enzymes: NADPH-dependent cytochrome-c-reductase, pseudocholinesterase and glucose-6-phosphatase. The substrate of the Odemethylase was p-nitroanisol.

The healthy human liver contains only $11 \pm$ 3 nmole cytochrome P-450/g liver wet weight in contrast to the rat liver with $30 \mathrm{nmole} / \mathrm{g}$ liver.

The cases of mild and medium severe hepatitisbased on clinical, chemical and histological criteria - showed no significant abnormalities in P-450 content and the activities of the other investigated enzymes, whereas severely diseased liver showed significantly decreased P-450 content accompanied by a lowered O-demethylation of p-nitroanisol, but with an unaltered NADPH-dependent cytochromec-reductase activity. A concomitant fall of pseudocholinesterase also signifies the injury of the endoplasmic reticulum. On the other hand the liver in patients treated with inducing drugs showed a threefold increase of P-450 content and a nearly twofold 
increase of NADPH-dependent cytochrome-creductase activity.

In all patients investigated the glucose-6-phosphatase was the only enzyme the activity of which did not change.

\section{1}

\section{Effect of Rifampicin, Isoniazid and} Streptomycin on the Human Liver:

\section{A Problem of Enzyme Induction}

\section{J. HAKIM, G. FELDMANN, J. BOUCHEROT, P. BOIVIN, P. GUIBOUT, and B. KREIS \\ Département d'Hématologie, and Unité de \\ Recherches de Physiopathologie Hépatique, \\ Hôpital Beaujon, 92-Clichy, and \\ Centre de Pneumophtisiologie, \\ Hôpital Cochin, 75-Paris, France}

It is well established that Rifampicin blood concentration decreases progressively to about $75 \%$ of the initial level, in patients treated with this drug. An hepatic microsomal enzymatic induction could account for the increased catabolism of the drug.

To test this hypothesis, 13 adults with pulmonary tuberculosis were studied by: (1) liver function tests including plasma sulfobromophthalein disappearance rate, total and unconjugated bilirubinaemia, serum transaminases and alkaline phosphatase activities, (2) hepatic bilirubin glucuronyl transferase activity (B-GTA) and paranitrophenol glucuronyl transferase activity (PNP-GTA), (3) optical and electron (four cases) microscopic studies. The results obtained after one month of treatment with Rifampicin ( $20 \mathrm{mg} / \mathrm{kg} /$ day), Isoniazid ( $15 \mathrm{mg} / \mathrm{kg} /$ day) and Streptomycin (1 g/day) were compared with the initial data found in each patient before treatment.

It was observed: (1) no change of liver function tests, of B-GTA and of the histological aspect on optical microscopy, (2) a mean increase of PNP-GTA of $50.5 \pm 27.54$ (SD) $\mu \mathrm{M}$ per hour and per $g$ of wet wt. liver $(P<0.001)$. The mean level of PNP-GTA before treatment was $29.0 \pm 14.82$ and after treatment was $79.5 \pm 27.5 \mu \mathrm{M} / \mathrm{h} / \mathrm{g}$ of wet wt. liver, (3) a proliferation of the smooth endoplasmic reticulum (SER) on electron microscopy in all the patients studied.

In conclusion: (1) Patients treated with Rifampicin, Isoniazid and Streptomycin have an hepatic SER proliferation and an increased PNP-GTA. Preliminary studies suggest that Rifampicin accounts for these effects. However, it may be that these effects could explain the increased hepatic catabolism of $\mathrm{Ri}$ - fampicin, (2) this potent microsomal enzymatic induction involving PNP-GTA and not B-GTA provides further evidence in favour of, at least, two glucuronyl transferase systems, (3) the different effect obtained on the two microsomal enzymatic activities studied can be due either to a 'toxic' effect on B-GTA equal to the induction effect or to a selective induction of a group of enzyme activities under a given inducer. The knowledge of the microsomal enzymatic activities enhanced by different inducers may provide a new classification of the drugs.

\section{2}

\section{Environmental Exposure to Organochlorine Pesticides and Hepatic Enzyme Induction in Man}

J. HUNTER, J. D. MAXWELL, D. A. STEWART, R. WILLIAMS, J. ROBINSON, and A. RICHARDSON The Liver Unit, King's College Hospital, London, S.E.5, and Shell Research Laboratory, Sittingbourne, Kent

Organochlorine pesticides are known to be potent hepatic enzyme inducing agents in animals but little is known about the effect of environmental exposure to these agents in man. Using the urinary excretion of D-glucaric acid as a measure of hepatic enzyme induction, we examined urine samples from workers at an organochlorine pesticide factory in Rotterdam. Mean D-glucaric acid excretion was $10 \cdot 1$ in seven office workers, 13.5 in 10 men in the aldrin/dieldrin plant and 27.3 in 10 men manufacturing endrin. These values are to be compared with mean levels of 8.0 in 30 controls and of 45.0 in 28 epileptics on a variety of anticonvulsant drugs; all values are expressed as $\mu \mathrm{M} / \mathrm{g}$ creatinine.

Urine samples and blood DDT levels were also estimated in a patient with severe unconjugated hyperbilirubinaemia before and during therapy with $30 \mathrm{mg} \mathrm{pp}{ }^{\prime}$ DDT daily. D-glucaric acid excretion began to increase and the plasma bilirubin to fall after three weeks when the blood DDT level had reached $0.203 \mu \mathrm{g} / \mathrm{ml}$.

A comparison of blood levels with D-glucaric acid excretion suggested that the level of exposure to DDT and similar compounds in Europe at present is unlikely to produce marked induction of hepatic enzymes - at least of those concerned in the glucuronic acid vathway. The effect of such agents is generairy less than that produced by anticonvulsant drugs in patients with epilepsy. 
Wellcome Research Laboratories Group,

Sucrose Gradient Centrifugation of Hepatitis Associated Antigen (H.A.A.) and Epidemic Hepatitis Associated Antigen (E.H.A.A.)

\section{A. BIANCHI-BOSISIO RIGHETTI,* P. A. BIANCHI, ${ }^{*}$ D. COSTANTINO, $\dagger$ \\ S. DEL PRETE, $\uparrow$ and M. DOGLIA $\dagger$ \\ *Istituto Patologia Medica I, †Clinica Medica II, University of Milano, Italy}

Centrifugation of H.A.A. has been previously described and sedimentation coefficients of 5OS$110 \mathrm{~S}$ have been reported.

In agreement with Bayer et al. when $0.2 \mathrm{ml}$ of H.A.A. positive serum was layered over a $10-30 \%$ sucrose gradient and centrifuged for $2.5 \mathrm{~h}$ at 125,000 $x \mathrm{~g}$ the Antigen was detected by immunoprecipitation electrophoresis in a narrow peak between fraction 17 and 22 (total fraction number $=30$ ).

Recently Del Prete et coll. identified a new antiserum which reacts not only with H.A.A. but also with an antigen (E.H.A.A.) present in the serum of patients affected by epidemic hepatitis.

To obtain more information on the physical properties of this antigen we have performed other centrifugation experiments using E.H.A.A. positive sera.

Unlike H.A.A., E.H.A.A. presented a much lower sedimentation coefficient (positivity was detected in fractions 20-30 of the gradient). Only when the same sera were centrifuged for 5 hours in $5-20 \%$ sucrose gradient, positive reactions were obtained between fractions 10 and 16.

The sedimentation coefficient of E.H.A.A., calculated using albumin as internal marker (4.41S), ranged from 11 to $14 \mathrm{~S}$.

These experiments suggest that H.A.A. and E.H.A.A. differ markedly in regard to their molecular properties.

\section{4}

\section{A Controlled Study for the Detection of Australia Antigen in the Urine}

\author{
R. A. FOX, F. J. DUDLEY, and S. SHERLOCK \\ Department of Medicine, Royal Free Hospital, \\ London, WC1X 8LF \\ K. APOSTOLOV, D. J. BAUER, and \\ J. W. SELWAY
}

Beckenham

Viruria is common in many viral infections, and might be expected in viral hepatitis. Urine has transmitted viral hepatitis on a few occasions ${ }^{\mathbf{1}, 2}$ and if infective, is probably only weakly so. Australia Antigen is a marker of one type of viral hepatitis, and we have looked for this in the urine of patients with and without the antigen in the serum. Each urine was examined, under code, for Australia Antigen, by gel diffusion, counter electrophoresis and complement fixation tests. All specimens were free of protein and blood.

In acute viral hepatitis Australia Antigen was found in the urine of eight of the 13 patients who had Australia Antigen in the blood. It was not detected in any of the nine patients with Australia Antigen negative viral hepatitis. These groups were comparable in length of disease and severity. In chronic liver disease the results were less clear cut. Four of 13 with Australia Antigen positive chronic liver disease had the Antigen detected in the urine. Three of 13 patients with Australia Antigen negative chronic liver disease were found to be positive in the urine. The patient with the highest titres of Australia Antigen in the urine was a patient with primary biliary cirrhosis who had been multiply transfused and had very high titres of serum antibody to Australia Antigen. The implications of this observation will be discussed.

Two of the seven normal subjects were positive for the Antigen in the urine. Both of these individuals were working in a laboratory investigating Australia Antigen.

Australia Antigen is frequently found in the urine of subjects with Australia Antigen positive liver disease and might be important in the transmission of the disease.

${ }^{1}$ Findlay, G. M., and Willcox, R. R. (1945). Lancet, 1, 212.

${ }^{2}$ Giles, J. P., Liebhaber, H., Krugman, S., and Lattimer, C. (1964). Virology, 24, 107.

\section{Persistence of Australia Antigen in Patients with Acute Viral Hepatitis}
J. O. NIELSEN, O. DIETRICHSON, P. ELLING, and P. CHRISTOFFERSEN
The Municipal Hospital, Department 2, and The Pathological-Anatomical Institute and Blegdams Hospital, The Department for Infectious Diseases, Copenhagen, Denmark 
Two hundred and fifty-three patients with biopsy verified acute viral hepatitis were examined for occurrence and persistence of Australia antigen (Au-antigen). For demonstration of Au-antigen an immunoelectroosmophoresis technique was employed according to Prince. At the time of admission Au-antigen was detected in sera from 112 of the patients $(44.3 \%)$. In sera from 88 patients Au-antigen appeared transitorily and the duration of antigenaemia varied from one to 13 weeks (average $4 \frac{1}{2}$ weeks). Thirteen patients are lost for control examinations. Au-antigen persisted for more than 13 weeks in 11 patients, and all of these developed clinical and biochemical signs of chronic hepatitis. In 10 of the 11 patients with persistent Au-antigenaemia, repeated biopsies showed development of chronic hepatitis. According to the classical histological features established by de Groote et al. eight patients had chronic aggressive hepatitis and two chronic persistent hepatitis. One patient refused a second biopsy; he showed clinical and biochemical signs of chronic hepatitis. Nine of the 11 patients with persistent Au-antigenaemia were males and two were females.

Fifty-nine of the 253 patients were examined with repeated biopsies three to 12 months after the first biopsy. Seventeen patients showed in these repeated biopsies development of chronic hepatitis. Ten of these had persistent Au-antigen, two had transitory Au-antigen in serum during the acute disease and five were females without any known inoculation and no Au-antigen was detectable in sera. Our conclusion is, that $4-5 \%$ of the patients with viral hepatitis admitted to Copenhagen hospitals had persistent Au-antigenaemia. All these patients developed chronic hepatitis.

'de Groote, J., Desmet, V. J., Gedigh, P., Korb, G., Popper, H.,
Poulsen, H., Scheuer, P. J., Schmid, M., Thaler, H., Uehlinger,
E., and Wepler, W. (1968). Lancet, 11, 626-628.
'Wright, R., McCollum, R. W., and Klatskin, G. (1969). Lancet, 11, 117-121.

16

\section{The Australia Antigen in Infantile Papular Acrodermatitis}

\author{
F. GIANOTTI \\ Clinica Dermatologica Dell 'Universita' di Milano \\ (Italia), 20122 Milano, Italy
}

Papular acrodermatitis (p.a.d.) is a common disease of children, which is widespread in every country and is presumably due to a still unknown virus. It is characterized by: (1) non-pruritic erythematopapular dermatitis on the face, buttocks and limbs lasting about 20-30 days; (2) a subcutaneous polyadenitis caused by reactive follicular hyperplasia; (3) a real acute hepatitis, rarely of the active chronic type, generally anicteric (icteric only in $5 \%$ of the cases).

The $\mathrm{Au}$ antigen was found in all the 33 children affected by p.a.d. who underwent examination. It generally appears 10-18 days after the onset of the dermatitis, and seldom after one or more months. It always remains present for more than one month, and generally for three to six months, either continually or intermittently; it can even last one or more years. This antigen can remain active at $-20^{\circ} \mathrm{C}$ (and $-80^{\circ} \mathrm{C}$ ) even for years.

The administration of prednisone and of azathioprine for a few days, while causing an immediate sharp reduction in SGOT and SGPT, does not modify HAA.

The continuous presence of HAA in p.a.d. seems to indicate the existence of a correlation with acute hepatitis, but not specifically with serum and acute infectious hepatitis, unless p.a.d. has the same origin as long-incubation hepatitis.

\section{7}

\section{The Relationship of Australia Antigen and Complement to Liver Injury}

\author{
F. J. DUDLEY, R. A. FOX, and S. SHERLOCK \\ Department of Medicine, The Royal Free Hospital, \\ London, WC1X 8LF
}

Both viral factors and the patients immune response have been suggested to be important in determining the clinical course following contact with Australia antigen. We have attempted to assess the importance of these factors by measuring the serum antigen titre, serum third component of complement $\left(C_{3}\right)$ and serum anticomplementary activity in 87 patients with antigen positive sera. Thirty-seven had acute hepatitis, 13 chronic persistent hepatitis ( $\mathrm{CPH}), 17$ chronic aggressive hepatitis (CAH) and eight primary liver cell carcinoma.

There was no correlation between Australia antigen titre and severity of the illness in acute hepatitis but in chronic liver disease titres were highest in the carriers, less in CPH and least in CAH and primary liver cell carcinoma. This inverse relationship between antigen titre and tissue damage is 
best explained by variations in either the humoral or cellular immune response.

Humoral immunity can produce tissue damage and antigen inactivation by the formation of immune complexes and this was assessed by measuring serum complement and anticomplementary levels. There was no relationship between either the serum anticomplementary levels or the third component of complement and the severity of liverinjury, except in fulminant hepatitis where complement was always low and similar to the levels found in fulminant hepatitis of different aetiology. This suggests that the low $\mathrm{C}_{3}$ levels in this group are due to decreased synthesis by the liver rather than increased consumption by immune complexes.

Our results suggest that the immune response to Australia antigen is important in determining the patient's clinical course and that the formation of immune complexes is not related to the degree of liver injury.

18

\section{Hepatitis-Associated Antigen and $\alpha$-Fetoprotein in Primary Liver-Cell Carcinoma in Greece}

ST. HADZIYANNIS, G. MERIKAS, A. AFROUDAKIS, and A. GUSTOSI Second Department of Medicine of Athens University, Hippokration General Hospital, Athens T.610-Greece

Recent studies indicate wide geographical variations in the incidence of hepatitis-associated antigen (HAA) in primary liver-cell carcinoma. In tropical areas and especially in Africa a high incidence (40\%) has been discovered whereas in Europe, the U.K. and U.S.A. the total number of reported positive cases is very limited (12 out of 235 patients).

In the present study 39 elderly Greek patients ( 34 males, five females, mean age of 63 years) with primary liver-cell carcinoma (34 with cirrhosis) were investigated for HAA (by counter-electrophoresis) and for $a$-fetoprotein (by Immunodiffusion). The incidence of $a$-fetoprotein has also been shown to vary widely.

HAA was present in 15 patients $(38 \%)$ and $a$-fetoprotein in $20(51 \%)$. Ten patients $(25 \%)$ had both tests positive and $14(35 \%)$ had both negative. HAA was not detected in the absence of underlying cirrhosis (five cases). Whereas $a$-fetoprotein was usually absent from patients with well-differentiated tumours the same was not true for HAA.

These findings accord with a high incidence of HAA positive viral hepatitis and chronic liver disease, discovered in Greece, and suggest that in this country, as in some tropical areas, the serologically demonstrable viral strain may be an important factor in the aetiopathogenesis of livercell carcinoma.

19

\section{Lecithin-Cholesterol-Acyl-Transferase (LCAT) Activity in Patients with Liver} Disease

H. GRETEN, H. WENGELER, and D. SEIDEL Medizinische Universitätsklinik, Heidelberg, Germany

Indirect evidence suggests that lecithin-cholesterolacyl-transferase (LCAT) is synthesized in the liver. There are, however, controversial reports in the literature correlating LCAT activity with various forms of liver disease. The purpose of this study was to determine LCAT activity in icteric patients which were classified not only by the conventional method, but also with respect to the presence or absence of LP-X, an abnormal plasma lipoprotein which specifically demonstrates or excludes cholestasis. With ${ }^{14} \mathrm{C}$ abelled cholesterol as substrate our enzyme assa y was (1) linear over $6 \mathrm{~h}$, (2) reproducible within $1 \%$ accuracy, (3) sensitive to measure even smallest amounts of LCAT activity in plasma. LCAT was measured at different stages of the disease and the patients divided into four groups: I. Hepatitis with cholestasis (LP-X pos.) (14); II. Hepatitis without cholestasis (LP-X neg.) (16); III. Extrahepatic biliary obstruction (LP-X pos.) (12); IV. Chronic liver failure (LP-X neg.) (10). Compared to normal controls (15), patients from group I had low, from group II had normal, from group III had normal and from group IV had low LCAT activity. In vitro studies clearly excluded circulating inhibitors of LCAT. Additional experiments demonstrated that LCAT is released or activated by human liver. These data explain contradictory findings on LCAT activity in liver disease by different investigators and suggest that combined determinations of LCAT and LP-X may prove useful as a means to differentiate not only between obstructive and non-obstructive jaundice, but also between extrahepatic biliary obstruction and intrahepatic cholestasis. 
Studies on the Characterization of the High- and Very-Low-Density-Lipoproteins in Patients with Liver Disease

\author{
D. SEIDEL, H. GRETEN, H. WENGELER, and \\ H. WIELAND \\ University of Heidelberg, \\ Medical Clinic (Ludolf-Krehl-Klinik), \\ 69 Heidelberg, Germany
}

It has previously been demonstrated that various liver disorders are associated with decreased concentrations of serum high density lipoproteins (HDL) and pre-beta-lipoproteins. However, not much information is available on the characteristics of these two classes of lipoproteins in liver disease. We herein report on studies designated to evaluate the possible mechanisms responsible for these altered lipoprotein patterns which are often found in acute hepatitis as well as in cholestasis and chronic liver failure. Evidence will be presented that the decreased concentration of HDL is primarily due to an impaired lipid binding capacity of Apo-A, the major protein moiety of HDL, resulting in an abnormally high protein/lipid ratio and a lack of neutral lipids in the HDL fraction. In contrast to normal alpha-lipoproteins, this fraction does not stain for lipids but shows two distinct precipitin bands on immuno-electrophoresis and on Ouchterlony plates. The isolated very-low-density-lipoprotein fraction (VLDL) from patients with liver disorders revealed a protein/lipid composition close to normal, but developed beta-mobility on electrophoresis. Analyses of the protein moieties of VLDL showed distinct differences compared to normal VLDL. While Apo-lipoprotein B and the characteristic VLDL peptides could be demonstrated, repeated analyses indicated a lack of Apo-lipoprotein A, which is a normal component of VLDL. This difference in protein composition is probably responsible for the altered electrophoretic mobility of VLDL in liver disease since it has been suggested that the pre-beta-mobility of normal VLDL derives from its Apo-A compound. Our data suggest that disturbed liver function leads to the synthesis of an abnormal Apo-A resulting (1) in an HDL dissociated into its two polypeptides and (2) in a VLDL lacking Apo-A. Both findings may be explained by the impaired capacity of this Apo-A to bind neutral lipids and may provide a better understanding of lipid metabolism in liver disease.

\section{Tetracycline-Induced Fatty Liver. Biochemical Mechanisms}

\author{
R. INFANTE, K. KOUMANOV, and J. CAROLI \\ Unité de Recherches de Physiopathologie \\ Hépato-biliaire, \\ U-9 de l'I.N.S.E.R.M., Hôpital Saint-Antoine, \\ 75-Paris XIIe, France
}

Hepatotoxicity of intravenous administered tetracyclines both in man and in animal is now well established. Histological findings include fatty liver and severe hepatic dysfunction.

The purpose of this work is to study the influence of tetracycline in the rat on lipid and plasma lipoprotein synthesis by the liver and on lipolysis of adipose tissue triglycerides.

Fasted rats received a single injection of tetracycline $(200 \mathrm{mg} / \mathrm{kg}$ body weight) and were sacrificed at different times. Liver protein synthesis and plasma lipoprotein release were studied in vivo by double labelling with ${ }^{3} \mathrm{H}$-palmitic acid and ${ }^{14} \mathrm{C}$-leucine. As early as $3 \mathrm{~h}$ after tetracycline administration a sharp decrease in plasma lipoproteins (VLDL, LDL, and HDL) concentration was observed. Fatty acid and aminoacid incorporation in plasma lipoproteins was dramatically depressed and VLDL virtually disappeared from plasma. Some recovery was noted $12 \mathrm{~h}$ after tetracycline injection.

Liver lipids progressively increased. At 3 hours a three-fold increase was observed in the concentration and the radioactivity of triglycerides thus reinforcing the idea of a lipoprotein release block.

Lipolysis of fat tissue triglycerides was inhibited after tetracycline injection, resulting in a fall in NEFA concentration. Furthermore, addition of the antibiotic in vitro into the incubation medium strongly inhibits the release of fatty acids from the fat pads.

22

\section{Rate of Albumin Synthesis in Enzymatically Isolated Rat Liver Cells}
K. WEIGAND, M. MÜLLER, J. URBAN, and G. SCHREIBER
Medizinische Universitäts-Klinik, Würzburg, and Biochemisches Institut der Universität, Freiburg, Germany

Isolated liver cells permit examination of the 
metabolism of intact cells in a medium free of hormones and metabolites circulating in the animal yet retain a greater degree of integrity than homogenates or cell-free systems.

Perfusion of the rat liver with collagenase and hyaluronidase yielded single liver cells with well preserved ultrastructure, as shown by electron micrographs. Cell suspensions were incubated for $90 \mathrm{~min}$ at $37^{\circ} \mathrm{C}$. The cells incorporated L-leucine- ${ }^{14} \mathrm{C}$ into protein at a constant rate for one hour. Between $8 \%$ and $15 \%$ of the added radioactivity $(2 \mu \mathrm{Ci} / \mathrm{ml})$ was incorporated into total protein after $90 \mathrm{~min}$. Incubation at $0^{\circ} \mathrm{C}$ served as control.

Albumin was purified by TCA precipitation, ethanol extraction, ammonium sulphate fractionation, gel filtration, ion-exchange chromatography, and polyacrylamid gel electrophoresis at $p \mathrm{H} \quad \mathbf{1 0 \cdot 3}$ and finally $p \mathrm{H} 2 \cdot 7$. This procedure yielded radiochemically pure albumin with a specific radioactivity of $4,650 \mathrm{dpm} / \mathrm{mg}$ for the $37^{\circ} \mathrm{C}$ incubation and $26 \mathrm{dpm} / \mathrm{mg}$ for the control. The ratio of albumin synthesis to total protein synthesis was $3.4 \%$, being the same as that observed in vivo. In contrast to a cell-free system, isolated liver cells maintain the specific function of albumin synthesis.

\section{3}

Localization of Albumin in Human Hepatocytes with Immunocytochemistry (Optical and Electron Microscopic Studies)

\author{
G. FELDMANN, J. PENAUD, and \\ J. CRASSOUS \\ Unité de Recherches de Physiopathologie Hépatique, \\ Hôpital Beaujon, 92-Clichy, France
}

Albumin synthesis by the hepatocytes is well established. The number of albumin-synthesizing hepatocytes, their localization within the lobule and the organelles responsible for this production are less well known. The investigation of these three points was the purpose of the present work. The material consisted of 15 surgical biopsies taken from patients without liver disease. Albumin was demonstrated by an immunocytochemical technique using human antibodies labelled with peroxidase. The excess free peroxidase was removed by filtration on Sephadex G 200. The same sections were used for optical and electron microscopic studies.

On optical microscopy, albumin was present only in part of the hepatocyte population $(35.9 \pm 13.5 \%$, $\mathbf{M} \pm \mathrm{SD}$ ). Albumin-containing cells seemed random- ly distributed within the lobule. The proportion of these cells was very different from one lobule to another one in the same specimen. The relatively small proportion of albumin-containing cells, at a given time, could be related to either intermittency or specialization of some hepatocytes in the synthesis of this protein. On electron microscopy, albumin was located exclusively in the granular and smooth endoplasmic reticulum: these findings are in agreement with previous biochemical work.

In conclusion: (1) in the normal human liver, at a given time, approximately one third of the hepatocytes contain albumin; (2) these albumin-containing cells are randomly distributed within the lobule; (3) inside these hepatocytes albumin is located in the endoplasmic reticulum, suggesting that these cells synthesize albumin.

24

\section{Changes in Ethanol Oxidation Rate in Liver Diseases and Endocrine Disorders in Man: Less Important than Dietary Factors and Drug Administration?}

H. GOEBELL, B. BUCHWALD, and Ch. BODE Department of Internal Medicine, University of Marburg, D-355 Marburg/L., Germany

Contradictory results have been published on changes in ethanol oxidation rate in man caused by different diseases (liver disease; hyperthyroidism; diabetes). Since marked alterations of ethanol oxidation have been observed in animal experiments by variation of the daily protein uptake and by drug administration we investigated in the present study whether similar changes occur in man.

In 25 healthy subjects and 65 patients with different diseases (liver cirrhosis; hyperthyroidism; diabetes mellitus) blood ethanol elimination rates (BEER) have been determined following i.v. infusion of $0 \cdot 3-0.4 \mathrm{~g}$ ethanol $/ \mathrm{kg}$ body weight by a sensitive enzymatic alcohol test.

\section{Results}

(1) In healthy volunteers BEER ranged from 1.4 to 3.9 (mean value $=2.47 \pm 0.79$ ) $\mathrm{mmoles} / \mathrm{l} / \mathrm{h}$.

(2) Variation of BEER from day to day were small $( \pm 2-10 \%$ ) when determined under identical conditions. 
(3) Administration of a protein deficient diet (20 g protein/day) to healthy volunteers for eight days decreased BEER significantly $(30-50 \%, P<0.01)$. After 'refeeding' a high protein diet (120 g protein/ day) for three days the ethanol oxidation capacity completely returns to initial values. The same effect was observed in patients with cirrhosis of the liver.

(4) Administration of several drugs (contraceptives, sedatives, antibiotics) for six to 10 days markedly increased BEER.

(5) In diabetics receiving no oral hypoglycemics the mean BEER was moderately elevated (3.24 \pm $0.53 \mathrm{mmoles} / \mathrm{l} / \mathrm{h}$ ) as compared to the controls $(2.47 \pm 0.79 \mathrm{mmoles} / \mathrm{l} / \mathrm{h})$. A similar elevation was found in hyperthyroidism (3.42 \pm 0.45 mmoles $/ 1$ /h). But patients of both groups received one to six different drugs for a longer period prior to study.

\section{Comment}

Protein deficiency decreases ethanol oxidation in man similar to that observed in animal experiments, where a marked fall in ADH-activity could be demonstrated. Since dietary manipulations and/or drug administration obviously affect BEER these factors should be considered when changes of BEER are found in certain diseases.

\section{Mechanism of Elevation of Alkaline Phosphatase (AP) and 5-Nucleotidase (5-NT) in Liver Obstruction}

\author{
A. BIANCHI BOSISIO RIGHETTI,* and \\ M. M. KAPLAN $\dagger$ \\ *Istituto Patologia Medica I, \\ University of Milano, Italy; \\ †New England Medical Center Hospital, \\ Boston, U.S.A.
}

5NT and AP are thought to measure the same parameter of liver dysfunction and are often used interchangeably during obstructive liver diseases.

A recent study showed that increase of serum AP in rats with bile duct ligation (b.d.l.) is due to de novo synthesis of this enzyme within the liver with subsequent leakage into serum.

Inhibition of protein synthesis and of RNA synthesis, one hour prior to b.d.l. prevents the increase of both hepatic and serum AP.

To elucidate the mechanism responsible for changes in serum $5 \mathrm{NT}$ activity in biliary obstruction, 40 rats underwent b.d.l. Half of them received cycloheximide one hour before ligation while others received normal saline; 16 rats were sham operated. Animals were sacrificed 0, 2, 5, 8 hours after b.d.l. 5NT and AP activity were measured in serum and dexoxycholate liver extracts.

5NT activity in control sera was very low with or without cycloheximide and increased 24-fold after b.d.l. This increase was prevented by cycloheximide only by $50 \%$.

Unlike AP, hepatic 5NT did not increase after b.d.l. and was not affected by cycloheximide.

The mechanism of 5NT serum elevation in liver disease is therefore quite different from that causing AP elevation and is more similar to the mechanism of elevation of GPT.

\section{6}

\section{Liver Removal Efficiency of Colloidal Radiogold in the Dog: An Approach using the Multiple Indicator Dilution Method}

\author{
C. BALABAUD, F. AFIFI, L. POINTARD, and \\ J-P. BENHAMOU \\ Unité de Recherches de Physiopathologie Hépatique, \\ Hôpital Beaujon, 92-Clichy, France
}

The uptake of colloidal radiogold $\left({ }^{198} \mathrm{Au}\right)$ during a single passage through the liver sinusoid can be assessed with the multiple indicator method. Using this approach, the authors have investigated three points: (1) is the removal efficiency (E) (i.e. the percentage of ${ }^{198} \mathrm{Au}$ taken up during a singlepassage) different when ${ }^{198} \mathrm{Au}$ comes to the sinusoid from either the hepatic artery (HA) or the portal vein (PV)? (2) Is E related to the transit time through the sinusoid? (3) What is the behaviour of ${ }^{188} \mathrm{Au}$ when its uptake is suppressed?

A mixture of ${ }^{51} \mathrm{Cr}$-labelled erythrocytes, ${ }^{125} \mathrm{I}$ labelled albumin and ${ }^{198} \mathrm{Au}$ was rapidly injected into either HA or PV. Blood samples, taken every second from the hepatic vein, were analysed for each of the indicators. The outflow concentrations of each were normalized by expressing them as a proportion of the total amount of each indicator injected per $\mathrm{ml}$ of blood. E was derived from the ratio of ${ }^{198} \mathrm{Au}$ dilution curve area to the 'non-uptake' indicator (erythrocyte, albumin) dilution curve area.

When the mixture was injected into HA or PV, E was respectively: $47.8 \pm 5.3 \%(M \pm S D)$ and 
$56.0 \pm 10.2 \%$; the difference is not significant (P $>0 \cdot 1)$. Erythrocyte sinusoidal mean transit time (erythrocyte ts), calculated using Goresky's model, was significantly correlated with $\mathrm{E}(\mathrm{r}=0.86, \mathrm{P}<$ $0.02)$. When a large dose of gelatin $\left(15 \mathrm{mg} / \mathrm{kg} \mathrm{b.w.} .^{-1}\right)$ was previously injected, E was not significantly different from zero; ${ }^{198} \mathrm{Au}$ ts can then be reasonably compared with the other 'non-uptake' indicators: ${ }^{198} \mathrm{Au}$ ts $(11.0 \pm 1.3 \mathrm{~s})$ was significantly longer than erythrocyte ts $(7.3 \pm 0.6 \mathrm{~s}, \mathrm{P}<0.02)$, but significantly shorter than albumin ts $(15.9 \pm 2.9 \mathrm{~s}, \mathrm{P}<$ $0.05)$. It is generally accepted that the longer albumin ts (compared to erythrocyte $\overline{t s}$ ) is due to the extra vascular diffusion of this indicator into the spaces of Disse; the shorter ${ }^{198} \mathrm{Au}$ ts suggests that the colloid diffuses only into a part of these spaces.

In conclusion: (1) ${ }^{198} \mathrm{Au}$ removal efficiency is similar from the hepatic artery and the portal vein; (2) ${ }^{198} \mathrm{Au}$ removal efficiency is related to the sinusoidal transit time; (3) ${ }^{198} \mathrm{Au}$ penetrates only into a part of the spaces of Disse.

\section{7}

\section{Hepatic Blood Flow and Liver-Scan Abnormalities in Cirrhosis}

Y. WEISS, D. MARLEAU, and C. SICOT

Unité de Recherches de Physiopathologie Hépatique, Hôpital Beaujon, 92-Clichy, France

Decrease in hepatic blood flow (H.B.F.) and extrahepatic uptake of colloidal radiogold $\left({ }^{198} \mathrm{Au}\right)$ on liver scan are frequently observed in cirrhosis. The purpose of this work was to study the relationship between these two abnormalities. In 27 patients with histologically proven cirrhosis, ${ }^{108} \mathrm{Au}$ liver-scan was performed with a scintillation camera; total H.B.F. was estimated using a constant infusion of indocyanine green (I.C.G.). In 12 patients, splenic and vertebral-marrow uptake was only faintly visualized; in this group of patients, H.B.F. was 1,353 \pm $332 \mathrm{ml} / \mathrm{min}(\mathrm{M} \pm \mathrm{SD})$; in 11 of them, H.B.F. was above $1,000 \mathrm{ml} / \mathrm{min}$. In the 15 other patients, splenic and vertebral-marrow uptake was more marked, i.e. slightly inferior or equal to hepatic uptake; in this second group of patients, H.B.F. was $730 \pm 132 \mathrm{ml} / \mathrm{min}$; in only one of them, H.B.F. was above $1,000 \mathrm{ml} / \mathrm{min}$. The H.B.F. mean value and the occurrence of H.B.F. higher than 1,000 $\mathrm{ml} / \mathrm{min}$ in these two groups of cirrhotics are very significantly different $(P<0.001)$. The present authors confirm there is no relation between extra- hepatic uptake of ${ }^{198} \mathrm{Au}$ and: $(a)$ the increase in wedge hepatic vein pressure $(P>0.20) ;(b)$ the decrease in the clearance $(P>0.70)$ and the extraction $(\mathrm{P}>0.80)$ of ${ }^{198} \mathrm{Au} ;(c)$ the decrease in the clearance $(P>0.20)$ and the extraction $(P>0.50)$ of I.C.G.

In conclusion, (1) H.B.F. is usually below 1,000 $\mathrm{ml} / \mathrm{min}$ in cirrhotics with marked extrahepatic uptake of radiogold, and above this level in patients with slight extrahepatic uptake; (2) these findings suggest that decrease in H.B.F. plays an important role in the increased extrahepatic uptake of radiogold, in cirrhosis.

\section{8}

\section{Plasma Clearance and Cerebral Effects of Chlorpromazine in Patients with Cirrhosis}

\author{
J. D. MAXWELL, S. CURRY, M. CARELLA, \\ D. PARKES, and R. WILLIAMS \\ Liver Unit, King's College Hospital, London, \\ S.E.5, and the Department of Pharmacology, \\ The London Hospital, London, E.1
}

Patients with cirrhosis may be unduly susceptible to the effects of sedative drugs, but it is still uncertain whether this is due to impaired drug metabolism or increased sensitivity of the central nervous system. Chlorpromazine has been recommended particularly in alcoholic cirrhosis, but its safety in chronic liver disease has recently been questioned by Read and Colleagues who showed that chlorpromazine produced electro-encephalographic slowing similar to that caused by morphine.

In this study plasma levels of chlorpromazine were measured for up to $\mathbf{4 8}$ hours following intravenous injection of a $25 \mathrm{mg}$ dose, in 24 patients with biopsy proven cirrhosis and in matched control subjects. A sensitive gas chromatographic assay was used which separated the drug from its metabolites. The plasma disappearance took the form of a double exponential decay curve. No difference between cirrhotic and normal subjects could be detected even when the effects of other drug therapy which might have caused increased activity of drug metabolising enzymes, was considered.

Drowsiness was experienced by all subjects and although a comparable increase in slow activity was found in both cirrhotics with and without previous encephalopathy, only in the latter group did encephalopathy become manifest after chlorpromazine. 
Thus the susceptibility of cirrhotic patients to chlorpromazine appears to be due to abnormal sensitivity of cerebral neurones rather than disordered drug metabolism.

\section{9}

\section{The Role of the Intestine in Experimental Acute Hepatic Encephalopathy}

\author{
J-P. PUJOL, D. PRANDI, J. ROCHE, \\ F. DEGOS, J-D. DEGOS, D. MARLEAU, \\ G. BLEICHNER, Y. WEISS, B. RUEFF, and \\ C. SICOT \\ (with the technical assistance of \\ D. BOURDIAUX and M. PEIGNOUX)
}

Unité de Recherches de Physiopathologie Hépatique, Hôpital Beaujon, 92-Clichy, France

The role of substances released from the intestine mainly ammonia, has often been considered in the mechanism of hepatic encephalopathy. The purpose of this work is to compare, in the rat, the electroencephalogram (EEG) after total hepatectomy and total evisceration (i.e., removal of the liver, digestive tract, pancreas and spleen). A three-stage procedure was used: $(a)$ ligation of the inferior vena cava above the renal veins; $(b)$ three weeks later, end-to-side portacaval anastomosis; and (c) two days after (by random selection), laparotomy alone (control group), hepatectomy (group A), or evisceration (group B). Animals from groups A and B were infused with glucose to maintain normal blood glucose level; insulin was added in group B. Rats were immobilized in a restraining cage and placed in a thermostatic room at $26^{\circ} \mathrm{C}$. In groups $\mathrm{A}$ and $\mathrm{B}$, animals which survived less than $11 \mathrm{~h}$ were excluded. Survival time was $16.25 \pm 2.30 \mathrm{~h}$ (mean $\pm 1 \mathrm{SD}$ ) in group $\mathrm{A}$ (10 animals) and $21 \cdot 30 \pm 2.50 \mathrm{~h}$ in group B (10 animals); the difference is significant $(P<0.01)$. The necropsy findings were similar in both groups, except for intestinal bleeding which was present in all the animals of group $\mathbf{A}$.

In groups $\mathbf{A}$ and $\mathbf{B}$, the EEG disorders were similar. They started 4-7 h after hepatectomy or evisceration. Their evolution was stereotyped: (a) decrease and disappearance of the spindles of high voltage spikes, normally present during the slow wave sleep; $(b)$ predominance of slow waves of 2-4 cps; and (c) fall in voltage and eventually a flat tracing. Blood ammonia was $5 \cdot 13 \pm 2.87 \mu \mathrm{g} / \mathrm{ml}$ in group $A$ and $1.03 \pm 0.68 \mu \mathrm{g} / \mathrm{ml}$ in group $B$; the difference is very significant $(P<0.001)$.
In conclusion, (1) the EEG disorders were similar in the hepatectomized and eviscerated rat; (2) these disorders cannot be related to blood ammonia which was high in the hepatectomized rat, but normal in the eviscerated animal; (3) the shorter survival time of the hepatectomized rat may be due to the intestinal bleeding in this group of animals.

30

Vinblastine Treated Primary Cultures of Rat
Liver: An Experimental Model for the
Detection of a Specific Enzyme Inducing
Action of Phenobarbital on in vitro
Hepatocytes

U. ARMATO, and P. G. ANDREIS

Department of Human Anatomy, Tissue Culture Laboratory, Medical School of the University of Padua, Italy

Vinblastine sulphate (VLB), a cytotoxic spindleinhibiting alkaloid, which is metabolized by the liver, was employed in a work aiming to demonstrate that primary cultures of differentiated mammalian liver cells can maintain at least some functional capabilities of liver tissue in vivo. VLB induced in cultured newborn rat and human adult liver cells $\mathrm{C}^{-}$, ball mitosis, cellular shrinking, appearance of huge crystals in the cytoplasm and of postmitotic multimicronuclei, and, furthermore, a dose-related degree of cell necrosis. The LD50 of VLB for rat liver cultures was employed as parameter of cellular enzymatic function. LD50 was $13 \mu \mathrm{g} / \mathrm{ml}$ after $24 \mathrm{hr}$ incubation. Following three days pretreatment of cultures with phenobarbital or $3^{\prime}-5^{\prime}$-cyclic-AMP (both $50 \mu \mathrm{g} / \mathrm{ml}$ ), LD50 changed to 115 and $90 \mu \mathrm{g} / \mathrm{ml}$ respectively. On the other hand, phenobarbital did not change significantly VLB LD50 for semicontinuous lines isolated from human embryo liver and lung, in contrast to the effect elicited in primary liver cultures, whereas 3'-5'-cyclic-AMP did. Thus phenobarbital acts on in vitro hepatocytes as a specific protective agent against VLB, whereas 3 '-5'-cyclicAMP acts as a non-specific one.

We believe we have shown that in vitro primary liver cultures behave as in vivo hepatocytes by responding to the enzyme inducer phenobarbital. 
This trial has been in progress for three years. To

A Comparative Study of Serum Immunoglubulins, Tissue Antibodies and Immunoglobulin-Containing Cells in the Liver

ST. HADZIYANNIS, A. MOUSSOUROS, and D. KARAMOUNTZOS

Second Department of Medicine of Athens University, Hippokration General Hospital, Athens Y.610, Greece

Although immunological changes have been extensively studied in liver disease it is still uncertain whether circulating autoantibodies in active chronic hepatitis $(\mathrm{ACH})$ and primary biliary cirrhosis (PBC) account for any elevation of serum immunoglobulins and whether immunoglobulin-containing cells (Igcells) in the liver, usually associated with high serum immunoglobulins (Hadziyannis, S., et al., 1969), may specifically contribute to autoantibody synthesis. In the present study serum immunoglobulins (IgG, IgA, IgM) and circulating autoantibodies (smooth-muscle, ANA and ' $M$ ') were measured in parallel with Ig-cells in the liver in various liver diseases (28) and miscellaneous non-hepatic conditions (26).

Comparison of fluorescent autoantibody titres of IgG, IgA and IgM class with respective serum immunoglobulin levels in $\mathrm{ACH}$ and $\mathrm{PBC}$ revealed no correlation.

High Ig-cells counts in the liver were seen in the absence of serum autoantibodies. In the presence of serum autoantibodies Ig-cells were usually present but there was no correlation between immunoglobulin classes of Ig-cells and of autoantibodies.

These findings suggest that serum autoantibodies do not account for any of the immunoglobulin changes in chronic liver disease and that Ig-cells frequently present in the liver of these patients do not appear to synthesize immunoglobulins with known autoantibody activity.

${ }^{1}$ Hadziyannis et al. (1969). Clin. Exp. Immunol., 5, 499.

32

A Controlled Trial of Azathioprine in Primary Biliary Cirrhosis

\section{A. ROSS and S. SHERLOCK}

Department of Medicine, The Royal Free Hospital, London, WC1X 8LF date, 17 patients have received $2 \mathrm{mg} / \mathrm{kg}$ azathioprine daily, and 19 control patients have been observed. Random allocation to either group has been used. Patients with overt liver failure, oesophageal varices or histological evidence of cirrhosis have been excluded from the study.

Complications of therapy have been slight-three treated patients have been withdrawn because of possible blood and bowel toxicity.

Pruritus has been relieved in five of the treated patients, and a significant improvement has occurred in serum levels of aspartate transaminase, cholesterol and alkaline phosphatase. Serum immunoglobulin M levels have also decreased with treatment, but there has been no consistent change in the titre of mitochondrial antibodies. Serial liver biopsies at yearly intervals have been compared, blindly, for cellular activity and fibrosis. Cellular activity was judged to have decreased in the biopsies of seven out of nine treated cases, and in one out of seven control cases. No consistent change in fibrosis was seen.

These findings suggest that azathioprine improves biliary excretion in primary biliary cirrhosis. Its effects on the long term prognosis of the disease are not yet known.

\section{Azathioprine and Corticosteroid Treatment of Hepatitis-Associated Antigen Positive Active Chronic Liver Disease}

ST. HADZIYANNIS and

A. EFSTRATOPOULOS

Second Department of Medicine of Athens University, Hippokration General Hospital. Athens T.610, Greece

The presence of hepatitis-associated antigen (HAA) in some cases of active chronic liver disease (ACLD) suggests a viral aetiology. Whether or not these 'viral' cases should be treated differently from those of the classic syndrome of active chronic hepatitis (as a rule HAA negative) is still unknown. In the present paper the response to azathioprine $(100 \mathrm{mg})$ and/or corticosteroids (10-15 mg) of 10 cases of HAA positive ACLD is described.

All patients had a long-standing chronic aggressive hepatitis type A (4) or B (6) which in four had progressed to cirrhosis. 
Before, during and following treatment serial determinations of HAA, serum immunoglobulins, tissue autoantibodies and liver function tests were performed.

Patients with established cirrhosis did not appear to respond either to azathioprine or to corticosteroids; several side-effects were also noted. In the remainder clinical and biochemical improvement was noted and in two (on azathioprine) function tests returned to normal. However, liver histology appeared to remain unchanged. In three patients HAA disappeared and in two its titre decreased. Disappearance of HAA was associated with appearance of smooth-muscle antibodies.

It is concluded that an early and cautious azathioprine or corticosteroid treatment of HAA positive ACLD appears to be justified but further controlled studies are necessary. The mode of action of these drugs is discussed.

\section{4}

\section{An Abnormal Bile Salt in Primary Biliary Cirrhosis}

G. M. MURPHY, F. H. JANSEN, and BARBARA H. BILLING

Department of Medicine, The Royal Free Hospital, Gray's Inn Road, London, WC1X 8LF

Bile acids were extracted from the sera of 15 patients with primary biliary cirrhosis, five patients with noncholestatic cirrhosis, and five normal subjects. The individual bile acid components were separated by thin layer chromatography and their concentration determined by reaction with hydroxysteroid dehydrogenase followed by fluorimetry. Unconjugated bile acids were not detected in any of the sera. No relationship was found between the concentrations of the individual bile acid conjugates and pruritus.

A conjugated bile acid which moved chromatographically in a similar manner to taurolithocholate was detected in the sera of 10 of the patients with primary biliary cirrhosis. It was not found in any of the sera from normal subjects or from the patients with non-cholestatic cirrhosis. Mass spectrometry of the free bile acid obtained after enzymatic hydrolysis showed that it was a monohydroxy unsaturated acidic steroid with a ring structure similar to that of cholesterol and therefore that it was not lithocholic acid.

The presence of conjugates of an unsaturated bile acid in the sera of patients with primary biliary cirrhosis suggests that in cholestatic liver disease there is an alternative pathway for bile acid formation.

\section{Relations between Bile Acid- and Adrenal Steroid Metabolism}

H. GERDES, K-P. LITTMANN, and W. DÖLLE Medizinische Universitätsklinik, 355 Marburg, Mannkopffstr. 1, Germany

It has been suggested ${ }^{1}$ that the altered steroid metabolism in cirrhosis is associated with intrahepatic cholestasis. Disturbed bile acid excretion seems to be an important feature of this condition. No data exists, however, on the correlation between steroid metabolism and bile-acid concentration of hepatic bile.

We investigated therefore in a group of patients with intra- and extrahepatic cholestasis the relation between the pattern of individual bile-acids and the biological half-life of cortisol. The main metabolites of cortisol were measured in the urine of the same group. The bile was obtained by transcutaneous puncture of intrahepatic bile-ducts. The individual bile acids and the $\mathrm{C}_{21}$-steroids were determined by gas chromatographic methods., ${ }^{2,3}$

\section{Results}

(1) In cholestasis there was a greatly reduced content of all bile acids in the hepatic bile.

(2) The trihydroxy : dihydroxy ratio of primary bile salts becomes reversed to less than 1 . The chief dihydroxy bile acid was chenodeoxycholic acid.

(3) Lithocholic acid could be identified in increased amounts when the concentration of chenodeoxycholic acid was especially high.

(4) There was an inverse relation between biological half-life of cortisol and bile acid content of hepatic bile.

(5) The pattern of cortisol metabolites was not significantly altered.

Slowed cortisol removal in cholestasis is an unexpected finding because there is no important biliary excretion and enterohepatic circulation of glucocorticosteroids. We assume, therefore, a disturbance of transport and/or binding of cortisol to the intracellular metabolic site.

\footnotetext{
1Zumoff, B. et al. (1968). J. clin. Endocr., $28,92$.

'Bailey, E. (1965). In: Lipsett, M.B. (ed.), Plenum Press, New York 'Nakayama, F. (1967). J. Lab. \& Clin. Med., 69, 594.
} 
on the M.P.values neither in the normal situation nor

\section{Measurement of Hepatic Cell Membrane Potential to Study the Effects of Drugs on the Liver with particular reference to Protection Against Anoxic Damage}

\author{
L. LAMBOTTE, J. B. OTTE, and \\ P. J. KESTENS \\ Laboratory of Experimental Surgery, \\ University of Louvain, Belgium
}

The measurement of hepatic cell membrane potential (M.P.) by means of intracellular microelectrodes gives precise information on membrane permeability and on transport of active ions; it can therefore be used for studying the mechanism of action of drugs on the liver. This approach has been made possible since perfusion of canine livers with blood maintains normal M.P. values for at least five hours. A reduction of the activity of the sodium pump results in a depolarization; for example, after the administration of ouabain there is a $20.9 \pm$ $1.9 \mathrm{mV}$ drop in 60 minutes. Similar results are also obtained after anoxia and in deep hypothermia.

With this new method of investigation, various drugs have been proved to modify the permeability of the hepatic cell membrane-for instance THAM increases the potassium permeability and therefore the M.P. On the other hand, chlorpromazine, which is supposed to stabilize the membranes, has no effect after an ischaemic period. Epinephrine produces a rapid but transient drop of potential $(-23.8 \mathrm{mV}$ $\pm 3.7 \mathrm{mV}$ in two minutes) attributed to an increased permeability for sodium. Results of practical importance have been obtained with isoproterenol, a $\beta$ sympathicomimetic agent which produces a significant hyperpolarization (from $45.7 \pm 1.5 \mathrm{mV}$ to $55.0 \pm 2.7 \mathrm{mV}$ ). This hyperpolarization is not dependant on active ion transport as it persists in presence of ouabain, but is related to a decreased sodium permeability. A reduction of permeability to sodium would diminish the energy requirement of the cell and could therefore protect the cells during anoxia. Effectively isoproterenol significantly improves the recovery of the M.P. after an ischemic period of 120 minutes at $20^{\circ} \mathrm{C}$ or of 180 minutes at $4^{\circ} \mathrm{C}$. Furthermore successful homotransplantation in four out of five dogs have been obtained after a three hours ischaemic interval with simple cooling of the liver with cold Ringer Lactate containing $0.2 \mathrm{mg}$ isoproterenol followed by storage in an ice-box. Tolerance to ischemia has not been extended before in dogs above two hours with simple cooling with normal electrolyte solutions.

\section{Conclusion}

Membrane potential measurement can be a new method for studying the mechanism of action of drugs on the liver. A practical result has been obtained with a demonstration of the beneficial effect of isoproterenol in hepatic transplantation. 